\title{
Una propuesta colaborativa para desarrollar el conocimiento profesional sobre la enseñanza inicial de la lectura
}

\author{
Luis Andrés CASTEJÓN FERNÁNDEZ \\ Soledad GONZÁLEZ-PUMARIEGA SOLÍS \\ Verónica MARTÍNEZ LÓPEZ
}

\begin{abstract}
Datos de contacto:
Luis Andrés Castejón

Fernández

Departamento de Psicología

Universidad de Oviedo

luiscf@uniovi.es

Soledad González-Pumariega Solís

Departamento de Psicología

Universidad de Oviedo

gsoledad@uniovi.es

Verónica Martínez López Departamento de Psicología Universidad de Oviedo martinezveronica@uniovi.es
\end{abstract}

Recibido: 13/06/2021

Aceptado: 26/07/2021

\begin{abstract}
RESUMEN
El conocimiento profesional -conjunto de representaciones que guían la práctica del profesorado en el aula y condicionan el rendimiento del alumnado- y el desarrollo profesional -recurso para propiciar el conocimiento profesional que facilita la puesta en marcha de prácticas instruccionales basadas en la evidencia, diseñadas con la finalidad de apoyar tanto la adquisición de conocimientos, habilidades y actitudes profesionales, como de su aplicación en la práctica- son componentes cada vez más relevantes en el ámbito de la enseñanza-aprendizaje, concretamente del lenguaje escrito. En este marco el modelo de respuesta a la intervención (RTI) representa una oportunidad, ya que, al poner en primer plano la calidad de la instrucción como herramienta para detectar y prevenir tempranamente el riesgo de una dificultad de aprendizaje, otorga gran valor al conocimiento profesional. La finalidad de este trabajo es presentar una propuesta de colaboración entre profesorado universitario y el profesorado de dos escuelas públicas con el objetivo de integrar en las aulas de Educación Infantil las evidencias sobre el aprendizaje lector y promover el conocimiento profesional. Participaron 75 aprendices de 3 은 de Educación Infantil, cuatro maestras y dos asesores universitarios. Se valoró el conocimiento profesional de las maestras, se identificó la diversidad del aula y el riesgo en el rendimiento lector. Se mejoró el conocimiento profesional del profesorado, así como su sistema de respuesta en el aula. Se redujo la diversidad inicial del alumnado y se redujo el riesgo de dificultades. La experiencia de colaboración ha funcionado y ha resultado satisfactoria para el profesorado.
\end{abstract}

PALABRAS CLAVE: Conocimiento profesional; Desarrollo profesional; Cooperación educativa; Instrucción basada en evidencias; Propuesta colaborativa. 


\title{
A collaborative proposal to develop professional knowledge about initial reading teaching
}

\begin{abstract}
Professional knowledge - a set of representations that guide teachers' practice in the classroom and impacts on student performance - and professional development - a resource to promote professional knowledge that facilitates the implementation of evidence-based instructional practices, designed with the purpose of supporting both the acquisition of knowledge, skills and professional attitudes, as well as their application in practice - are increasingly relevant components in the field of teaching-learning, specifically of written language. In this framework, Response to Intervention model (RTI) represents an opportunity since, by putting the quality of instruction in the foreground as a tool to detect and prevent early the risk of a learning difficulty, it places great value on professional knowledge. The purpose of this work is to present a proposal for collaboration between university staff and the teaching staff of two public schools with the aim of integrating evidence on reading learning in Early Childhood Education classrooms and promoting professional knowledge. Seventy-five students from the 3rd year of kindergarten, four teachers and two university advisers participated. The professional knowledge of the teachers was assessed, the diversity of the classroom and the risk in reading performance were identified. The professional knowledge of the teachers as well as their response system in the classroom improved. The initial diversity of the students was reduced, and the risk of difficulties was reduced. The collaboration experience has worked and been satisfactory for the teachers.
\end{abstract}

KEYWORDS: Professional knowledge; Professional development; Teacher collaboration; Evidence based practices; Collaborative proposal.

\section{Introducción}

El conocimiento profesional se refiere a las representaciones que guían la práctica del profesorado en el aula, cuyo estudio es relevante porque condiciona el rendimiento del alumnado (Didion et al., 2019; Evens et al., 2018). Schön (1998) lo caracterizó como un repertorio de acciones, un sistema de respuesta basado en experiencias previas desde las que el profesorado toma decisiones en la práctica. Un aspecto de gran interés es su naturaleza implícita y, dado que no se refleja en el discurso del profesor, necesita ser explicitado estratégicamente por los asesores (profesorado universitario) para conectar con el profesorado al iniciar una colaboración (Pozo y Gómez-Crespo, 2005; Sánchez y Mena, 2010; Scarparolo y Hammond, 2018; Schön, 1998).

El medio para propiciar el conocimiento profesional es el Desarrollo Profesional (DP). Este facilita la puesta en marcha en las aulas de prácticas instruccionales basadas en la evidencia, diseñadas con la finalidad de apoyar tanto la adquisición de 
conocimientos, habilidades y actitudes profesionales, como de su aplicación en la práctica (NPDCI, 2008).

El DP debe partir del nivel de conocimiento del profesorado. Una de las dificultades principales de los asesores en el ámbito del lenguaje escrito radica en acceder a ese conocimiento de la práctica (Sánchez y Mena, 2010). La literatura reconoce la frustración que esa conexión fallida ha generado en investigadores, asesores y profesorado que no terminan de encontrarse (Alegría, 2006; Mena et al., 2008; Sánchez, 2001; Snow et al., 2005). El asesoramiento constructivista ha insistido en solucionar esa dificultad considerando como punto de partida lo que el profesorado conoce, piensa, siente y hace cuando enseña la lectura en el aula. Se destaca así la necesidad de considerar qué motiva y compromete al profesorado para mejorar los procesos educativos implicados en sus rutinas instruccionales (Osman y Warner, 2020). Para ello se han utilizado metodologías etnográficas de observación en el aula, cuestionarios y procedimientos dialógicos sobre la práctica y sus dificultades (Jiménez et al., 2015; Sánchez y Mena, 2010; Sánchez et al., 2010).

En el campo concreto de la enseñanza-aprendizaje del lenguaje escrito, el conocimiento profesional, la motivación, la actitud y el DP son componentes cada vez más relevantes (Aaron et al., 2008; Arrow et al., 2019). Lane et al. (2009) encontraron que los aprendices de primer curso que tenían maestros con más conocimientos sobre la fluidez obtuvieron puntuaciones más altas en precisión y velocidad en la decodificación de palabras que aquellos alumnos cuyos profesores tenían menos conocimientos; de la misma manera, los aprendices de segundo curso obtuvieron mejores puntuaciones no solo en el reconocimiento automático de palabras, sino también en la lectura de pequeños textos. Es decir, lo que conoce el profesor repercute directamente en el rendimiento del alumnado (Didion et al., 2019).

El conocimiento profesional es, por tanto, un condicionante externo del rendimiento lector especialmente importante en los primeros momentos (Didion et al., 2019; Scanlon et al., 2008; Scanlon et al., 2005), ya que la limitación en la motivación y el conocimiento del profesorado en la fase inicial tiene un efecto negativo en el rendimiento a corto y largo plazo del alumnado (Acosta et al., 2008; Didion et al, 2019). A corto plazo, por la función mediadora del lenguaje escrito en el proceso de aprendizaje en distintas áreas de conocimiento (Aguilar et al., 2017; Pfost et al., 2014). $Y$ a largo plazo, al condicionar un futuro incierto para el alumnado. En nuestro contexto se ha relacionado el bajo rendimiento lector con la elevada tasa de Abandono Escolar Temprano, que en España llegó a un 17,9\% en 2018 (Acosta, et al., 2008). Afecta a la cualificación lograda por los aprendices, pudiendo abocarles al subempleo y en ocasiones a la delincuencia (Ou et al., 2007).

En este marco argumental el modelo de respuesta a la intervención (RTI) representa una oportunidad porque, al poner en primer plano la calidad de la instrucción para detectar tempranamente (Educación Infantil) el riesgo de una dificultad de aprendizaje, otorga valor al conocimiento profesional, a las rutinas del aula y al desarrollo profesional (Jiménez, 2019). Así, McCutchen et al. (2002) señalan que el incremento en el conocimiento de los profesores tiene efectos sistemáticos en sus prácticas instruccionales. El modelo RTI permite identificar al alumnado que está en riesgo de presentar una dificultad de aprendizaje, intervenir y hacerle un seguimiento en diferentes momentos del curso escolar. Este modelo respalda la prevención y la instrucción basada en la evidencia científica (Jiménez, 2019). El asesoramiento en esta etapa se ocupará de concienciar al profesorado sobre su labor 
preventiva, de explicitar su conocimiento profesional sobre los precursores y la enseñanza inicial de la lectura (Jiménez, 2019; Scanlon et al., 2008; Suárez-Coalla et al., 2014) y de motivarle y capacitarle para identificar y dar respuesta ajustada a la diversidad inicial (Arrow et al., 2019; Osman y Warner, 2020; Scanlon et al., 2008; Scarparolo y Hammond, 2018).

La finalidad de este trabajo es presentar una propuesta de colaboración entre profesorado universitario (asesores) y el profesorado de dos escuelas públicas con el objetivo de concienciarlo y capacitarlo para identificar y dar respuesta a la diversidad del aula y reducir el riesgo lector. De ese modo se difunden evidencias relacionadas con la adquisición de los procesos básicos de lectura, referidas tanto a los precursores (conciencia fonológica y denominación rápida) como a la elaboración de representaciones ortográficas (Castejón et al., 2015; Defior y Serrano, 2011; GutiérrezFresneda y Díez-Mediavilla, 2015; Suárez-Coalla et al., 2014).

Los objetivos específicos son: 1 . Contactar con las escuelas para establecer una colaboración Universidad-Escuela y constituir grupos de trabajo a nivel de centro. 2 . Explicitar el conocimiento profesional del profesorado. 3. Aplicar colaborativamente en el aula tareas de valoración de los precursores de la lectura y la identificación de palabras. 4. Establecer medidas de criterio (criterio 1) para elaborar el mapa lector del grupo clase identificando tres niveles lectores: riesgo, emergente y establecido. 5. Implementar propuestas basadas en evidencia para trabajar en el aula de manera sistemática los precursores de la lectura y la identificación de palabras. 6. Analizar conjuntamente el mapa de la clase posterior a la intervención a partir de los resultados de la evaluación final y del criterio establecido. 7. Identificar al alumnado en riesgo persistente y derivarlo a una evaluación específica. 8. Valorar el efecto de la intervención en la reducción del riesgo lector y determinar el patrón de compensación de la diversidad establecido con el criterio 1: efecto Mateo (efecto bajo), ejecución estable (efecto medio) o compensación de las diferencias (efecto alto). 9. Establecer un nuevo criterio (criterio 2) y el nuevo mapa lector para orientar el trabajo en el siguiente curso.

\section{Método}

\section{Participantes}

La muestra inicial para este estudio de tipo no probabilístico y carácter intencionado estaba formada por 71 participantes de tercer curso de Educación Infantil de dos escuelas públicas de una comunidad del norte de España. Del total de la muestra, 43 participantes eran niñas y 28 niños. La edad cronológica media era 64,3 meses (DT=3,158). En la Tabla 1 se recoge el número de participantes por cada Escuela en función del género, así como la media (M) y la desviación típica (DT) en edad cronológica. 


\section{Tabla 1}

Número de sujetos en cada Escuela por sexo y edad cronológica media (M) y DT

\begin{tabular}{lcccc}
\hline & \multicolumn{2}{c}{ Sexo } & & \multicolumn{2}{c}{ Edad cronológica } \\
\hline & Niños & Niñas & M & DT \\
\hline Escuela A & 9 & 20 & 65,62 & 3,448 \\
Escuela B & 19 & 23 & 63,45 & 2,634 \\
\hline
\end{tabular}

Las cuatro maestras de Educación Infantil (una por cada grupo de clase y dos grupos de clase por cada escuela) se integraron en Grupos de Trabajo (dos en total, uno por cada escuela implicada) con dos profesores universitarios como asesores. Esos grupos eran gestionados por el Centro de Profesores y Recursos de la Comunidad Autónoma y contaban con el apoyo de los equipos directivos de los centros.

\section{Instrumento}

Los asesores universitarios seleccionaron, organizaron y presentaron al profesorado seis tareas para valorar con el grupo de clase la denominación rápida, la conciencia fonológica y la lectura de palabras (González-Seijas y Cuetos, 2008; Jiménez y Ortiz, 1995; Sellés et al., 2010).

La primera tarea consistió en denominar rápidamente una lista de 36 letras escritas en mayúsculas (RLA), incluía vocales y consonantes. En la segunda tarea, de conciencia fonémica (CFI), el sujeto tenía que identificar el sonido inicial de 10 palabras, dos monosílabas y ocho bisílabas (seis tienen la estructura CVCV y dos CCVCV). La instrucción fue ¿Cuál es el primer sonido de estas palabras? En la tercera tarea, también de conciencia fonémica (CFD), el sujeto tenía que reconocer auditivamente qué palabra de un grupo de tres empezaba por un fonema diferente. Estaba formada por 10 grupos de tres palabras bisilábicas, donde los cinco primeros grupos tenían la estructura CVCV y los cinco últimos la estructura CCVCV. La instrucción fue ¿Cuál de estas palabras comienza con un sonido distinto? La cuarta tarea consistió en la lectura de 20 palabras (LPA) cortas bisílabas de alta frecuencia relacionadas con el vocabulario curricular, 17 tenían la estructura CVCV y tres la estructura VCCV. La quinta tarea consistió en la lectura de 10 pseudopalabras (LPSA), tres monosílabas, seis bisílabas de estructura CVCV y una de estructura VCV. En estas dos últimas tareas la instrucción fue "Lee lo más rápido que puedas estas palabras". La sexta tarea consistió en la denominación rápida (RIT) de 20 estímulos (nueve imágenes que se repiten) seleccionados por las maestras y conocidos por el alumnado (figuras geométricas, números y dibujos), todos ellos en blanco y negro. La instrucción fue "Di lo más rápido que puedas el nombre de estos dibujos" (González-Seijas y Cuetos, 2008; Jiménez y Ortiz, 1995; Sellés et al., 2010).

Estas tareas sirvieron para afianzar el conocimiento profesional de las maestras y, a partir de medidas de criterio establecidas colaborativamente, para identificar los niveles de rendimiento en las aulas en el pretest y postest (objetivos 4, 6, 7, 9). 


\section{Procedimiento}

El contacto con las escuelas se produjo en el marco de una formación de inicio de curso escolar organizada por la Consejería de Educación, a la que asistió voluntariamente profesorado de Educación Infantil y Primer Ciclo de Primaria. La presentación por parte de dos profesores universitarios de las consecuencias del riesgo inicial en el aprendizaje de la lectura y del modelo RTI despertó el interés de un grupo de docentes de distintos centros para colaborar (objetivo 1).

La colaboración con las maestras de Educación Infantil comenzó en dos centros y con el fin de acceder al conocimiento profesional de las profesoras participantes, los asesores universitarios plantearon un diálogo con tres preguntas abiertas: 1. ¿Qué haces cuando enseñas a leer en el aula de 3o de Educación Infantil y cuánto tiempo dedicas a esas actividades? 2. ¿Qué dificultades encuentras en la enseñanza de la lectura? 3. ¿Te sientes competente para la enseñanza inicial de la lectura? Con la colaboración del coordinador del grupo (una maestra de cada centro) se registraron por escrito las opiniones y el sentir del profesorado. Finalizada la sesión, la coordinadora y el asesor contrastaron sus notas recogiendo lo más relevante en un acta que en la siguiente reunión fue confirmada por el profesorado participante (objetivo 2).

Posteriormente los asesores presentaron, justificaron, concretaron con ejemplos y confrontaron con las profesoras las seis tareas descritas en el apartado anterior (objetivo 3). Una vez aplicadas en sus aulas en el mes de octubre, los resultados de la evaluación inicial sirvieron para establecer una medida de criterio útil para definir el mapa lector, diferenciando los niveles "riesgo", "emergente" y "establecido" (Good et al., 2001; Samuels, 1979) (objetivo 4).

A continuación, los grupos de trabajo se focalizaron en el objetivo quinto, mejorar el mapa lector inicial y reducir el riesgo lector en el grupo clase. Para ello se determinaron las necesidades educativas de cada grupo y de cada aprendiz en cada uno de los bloques (RAN, conciencia fonológica y lectura de palabras y pseudopalabras) y se diseñaron colaborativamente las medidas organizativas y las actividades necesarias para dar respuesta desde el aula a las dificultades detectadas. Entre la evaluación inicial y final (siete meses) el profesorado desarrolló regularmente las actividades diseñadas. Quincenalmente se revisaban en grupo.

Para alcanzar el sexto objetivo se realizó la evaluación final durante el mes de mayo de ese curso académico y se compartieron los resultados con el profesorado. Se presentaron los patrones de adquisición de la lectura para constatar si se había superado el Efecto Mateo, se había instaurado un patrón de ejecución estable o un patrón de compensación (Pfost et al., 2014). Finalmente se discutieron los resultados, las debilidades y fortalezas de la propuesta, así como su continuación y posible expansión a otros centros.

\section{Análisis de datos}

Se realizó un diseño cuasiexperimental pretest-postest para un solo grupo. Los análisis estadísticos fueron realizados con el programa estadístico SPSS versión 25.0 para Mac y el programa G*Power 3.1. Con el primero se obtuvieron los estadísticos 
descriptivos correspondientes. Se calculó el índice de fluidez léxica (IFL) para cada participante. Este índice se obtiene del número total de palabras leídas correctamente dividido por el tiempo invertido en leerlas y todo ello multiplicado por 100. Además, se llevó a cabo un análisis factorial (ANOVA) y la prueba post-hoc de Scheffé para conocer si el IFL servía para establecer el continuo y si había diferencias entre los niveles de lectura. Se realizó un análisis de correlación de Pearson entre las variables precursoras de la lectura con la precisión y la velocidad lectora. También se utilizó la prueba $t$ de Student para determinar si había diferencias estadísticamente significativas antes y después de la intervención y se obtuvo el cálculo post-hoc de la potencia observada y el tamaño del efecto con el programa G*Power 3.1 para valorar la efectividad de la propuesta de intervención en cada una de las variables y para compensar la ausencia de grupo control. Además, se empleó el índice $d$ de Cohen (Cohen, 1988) para medir el tamaño del efecto, siendo un índice que pertenece a la familia de índices que miden la magnitud del efecto del tratamiento. Es una de las medidas más empleadas para el cálculo de la potencia del efecto (Ledesma et al., 2008) que establece efectos pequeños cuando se sitúa alrededor de 0,20, efectos medianos si está próximo a 0,50 y efectos altos cuando se sitúa en torno a 0,80 (Cohen, 1988).

\section{Resultados}

Los resultados se exponen siguiendo la secuencia de objetivos.

1. Características del conocimiento del profesorado: qué conoce y hace, qué dificultades encuentra y qué percibe el profesorado sobre la enseñanza de la lectura.

En cuanto a la cuestión qué conoce y hace, el profesorado manifestó conocer y enseñar con eficacia en el aula la conciencia fonológica de forma paralela a la enseñanza del principio alfabético. De los comentarios del profesorado se dedujeron dos limitaciones: primera, cierta inconsistencia en la base conceptual a la hora de diferenciar los niveles de la conciencia fonológica (silábica, intrasilábica y fonémica); y segunda, la falta de estructura y sistematicidad en la aplicación práctica de dichos niveles y en las actividades que se realizan en cada nivel (identificar, omitir, añadir...). Las actividades de conciencia fonológica resultaron menos organizadas y sistemáticas que las orientadas a la enseñanza del principio alfabético y ello debido a que las maestras asumían actividades de distintos programas que adaptaban al aula tal cual.

Además, la denominación rápida como precursor era desconocida por el profesorado. Se relacionaba más con el aprendizaje del vocabulario que con la fluidez. Tampoco reconocían la fluidez lectora (precisión, velocidad y prosodia) como contenido de la enseñanza ni advertían la dificultad que representa en el aprendizaje de la lectura. Este hecho contrastaba con la facilidad con que adquirían el contenido al que dedicaban más tiempo, la precisión (Castejón et al., 2015; Suárez-Coalla et al., 2014). Respecto a la automatización de los procesos básicos, el profesorado no trabajaba de manera sistemática y específica este aspecto.

Acerca de la cuestión ¿qué haces cuando enseñas a leer en el aula?, el profesorado estaba centrado en la enseñanza sistemática del principio alfabético, organizada en torno a las correspondencias entre grafemas y fonemas. Seguían una secuencia 
ordenada y con cada fonema incorporaban la forma y el nombre del grafema, un gesto de apoyo y una palabra (es la "efe" y suena /f/ para decir FOCA). Después elaboraban fichas con listas de palabras y frases sencillas que el alumnado leía. Para el profesorado leer era, principalmente, aplicar el principio alfabético con exactitud, sin errores. Se obviaban la elaboración de representaciones ortográficas y la fluidez lectora.

En cuanto a los procedimientos, no utilizaban sistemáticamente la metodología de lecturas repetidas de palabras que es clave para adquirir la fluidez. Más aun, manifestaban cierta reticencia a repetir la lectura de listas de palabras por el efecto negativo que esa práctica creían que podría representar para la motivación y la comprensión ("no les puedo tener leyendo siempre lo mismo", "así no se van a aficionar a la lectura, tienes que pensar en eso", "leer no es leer rápido es comprender").

En relación a la segunda cuestión, ¿Qué dificultades encuentras en la enseñanza de la lectura?, el profesorado refería dificultades de carácter general como la falta de madurez del alumnado para aprender a leer, la pobreza de vocabulario, la falta de colaboración de las familias y, especialmente, la diversidad del alumnado("cada vez son más inmaduros", "tienen poco vocabulario", "hay familias y familias", "se distraen continuamente", "no leen en casa", "cómo van a aprender a leer algunos, casi no saben hablar", "no hablan con ellos, los dejan con la Play", "no tienen libros en casa" "no les leen cuentos", "son lectores de pantallas").

Y en cuanto a la tercera cuestión, ¿Te sientes competente para la enseñanza inicial de la lectura?, las profesoras manifestaban una percepción de competencia positiva -se sentían competentes- pero al mismo tiempo expresaban necesitar formación para identificar y atender a la diversidad de niveles del aula ("cada curso es más difícil enseñar a leer"). Un resultado interesante para el DP es que no percibían incompetencia por las lagunas identificadas en cuanto a la denominación rápida y la fluidez lectora. Tampoco eran conscientes de la limitación conceptual identificada respecto a la conciencia fonológica (p.e. dificultades para diseñar actividades de conciencia fonémica) ni de la falta de sistematicidad y organización en este trabajo (en cuanto a niveles y actividades). Es más, entendían que este contenido estaba bien atendido y que ya tenía un efecto importante en el rendimiento lector de su alumnado.

\section{Identificar la diversidad inicial en el aula de 3ํ de Educación Infantil.}

Como se puede observar en la Figura 1, la distribución de los participantes de los dos grupos tomando como variables el tiempo (LPT) y el número de aciertos en lectura de palabras (LPA) era muy heterogénea y diversa (cada punto representa a un participante). 


\section{Figura 1}

Distribución inicial de los sujetos en función del tiempo y lectura correcta de palabras

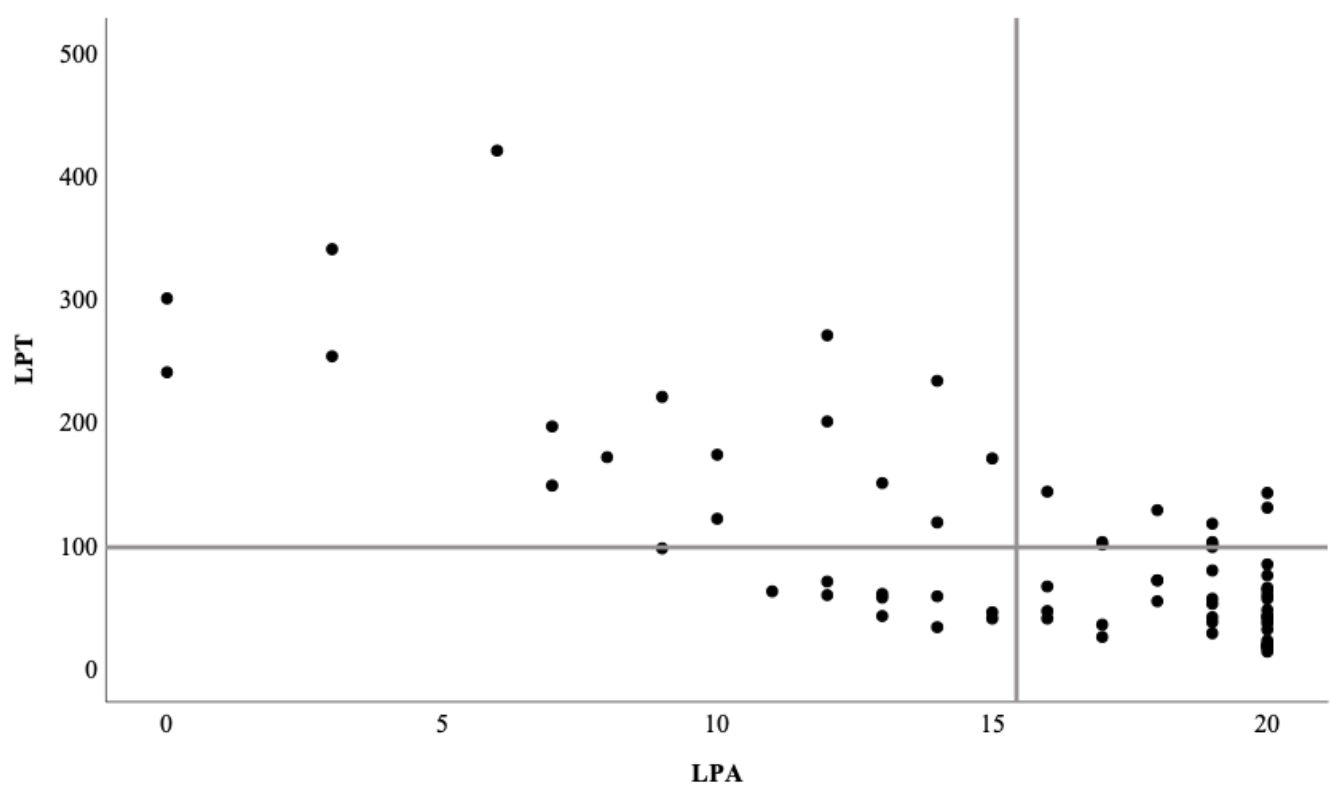

En la Figura 1, tal como se presentó en los grupos de trabajo, se refleja la diversidad indicada por las maestras como principal dificultad de enseñanza (las palabras que leen correctamente se ven la línea vertical y los tiempos empleados en la horizontal). En la Tabla 3 se recogen los estadísticos descriptivos de todas las tareas antes de poner en marcha la propuesta de intervención.

3. Establecer la medida de criterio para identificar los niveles ("riesgo", "emergente" $y$ "establecido") del grupo.

En los grupos de trabajo se planteó qué medida se debería establecer como criterio para delimitar los niveles del grupo (riesgo-emergente-establecido) y poder ajustar la respuesta educativa del aula a cada uno. El profesorado optó por la lectura de palabras que es resultante de las habilidades precursoras (Castejón et al., 2015; Suárez-Coalla et al., 2014). Finalmente se acordó utilizar un índice que combina precisión y velocidad: el Índice de Fluidez Lectora (IFL) (Valencia et al., 2010).

Antes de establecer los valores críticos o de corte se eliminaron del total de puntuaciones aquellos valores extremos en la variable tiempo de lectura de palabras y de cuatro participantes que no completaron las pruebas por diversos motivos, como el cambio de centro o la ausencia prolongada al aula. Aquellos participantes cuyo tiempo estaba por debajo del percentil $20(6,001)$ deberían formar parte directamente del nivel de Riesgo y no se utilizarían para calcular la medida de corte. Una vez eliminados, se calculó el criterio 1 a partir de la media $(40,02)$ y la desviación típica $(32,404)$ del IFL para identificar los niveles en cada grupo de clase. 
Se estimó que aquellos participantes cuyas puntuaciones estaban una desviación típica por debajo de la media (M-1DT) formarían también parte del nivel de Riesgo (< $7,62)$. Los que se encontraban una desviación típica por encima de la media (M+1DT) formarían el nivel Establecido $(>72,43)$. Todos aquellos cuyas puntuaciones se encontraban entre los dos valores anteriores formarían el nivel Emergente (entre 72,42 y 7,63). En la Tabla 2 se observa el número de sujetos que por su puntuación estaban en cada uno de los grupos, así como la media y la desviación típica del IFL de los grupos antes de la intervención.

\section{Tabla 2}

Número de sujetos de cada grupo (n), media (M) y la desviación típica (DT)

\begin{tabular}{lccc}
\hline Niveles & $\mathrm{n}$ & $\mathrm{M}$ & $\mathrm{DT}$ \\
\hline Establecido & 7 & 116,41 & 19,612 \\
Emergente & 46 & 32,21 & 14,860 \\
Riesgo & 18 & 4,94 & 3,391 \\
\hline
\end{tabular}

El ANOVA de un factor entre niveles en la variable IFL antes de implementar la propuesta muestra efectos significativos en los tres niveles $(F(2,68)=171,0831$; $\mathrm{p}<0,001)$. En la prueba post hoc de Scheffé se observa que las diferencias estadísticamente significativas se mantenían entre el nivel Establecido y el Emergente $(p<0,001)$, entre el Establecido y el Riesgo $(p<0,001)$, y entre el nivel Emergente y el de Riesgo $(\mathrm{p}<0,001)$.

Cada aprendiz fue asignado a un grupo y se diseñó colaborativamente un entrenamiento en los contenidos en que obtuvo puntuaciones bajas: conciencia fonológica y/o denominación rápida y/o lectura de palabras. El seguimiento se realizaba en el aula y, cuando se consideraba necesario, se requería el apoyo de otro profesor (bien la maestra de pedagogía terapéutica, bien la maestra de audición y lenguaje que participaba en el aula) o de la familia. En las reuniones quincenales del grupo de trabajo se comentaba la evolución de los aprendices y se revisaban las actividades llevadas a cabo.

Para reforzar el conocimiento del profesorado sobre el modelo que relaciona los dos precursores, conciencia fonológica y denominación rápida, se obtuvieron las correlaciones de esos valores con la precisión lectura y la velocidad lectora. Se corroboró el modelo al ser significativa la correlación entre las variables LPA y CFI $(r=0,609 ; p<0,01)$, LPA y CFD $(r=0,493 ; p<0,01)$, LPT y RLA ( $r=-0,317 ; p=0,007)$, LPT y CFD ( $r=0,362 ; p=0,002)$ con el total del grupo.

4. Resultados del entrenamiento de acuerdo con el criterio inicial: nuevo mapa lector de cada grupo.

En la Tabla 3 se muestran los resultados obtenidos en todas las variables estudiadas antes (PRE) y después (POST) de la aplicación de la propuesta de intervención, el valor del estadístico $t$, su significación, la potencia observada y el tamaño del efecto. En las variables en las que se computan los aciertos, los 
participantes obtuvieron puntaciones medias superiores, mientras que en las que se computa el tiempo, las puntuaciones fueron menores en POST que en PRE. En todas ellas hay diferencias estadísticamente significativas, pero es en CFI, CFD, LPA, LPT, LPSA y IFL donde se observó un mayor progreso, siendo el tamaño del efecto alto en las variables CFD $(d=1,000)$ y LPA $(d=0,803)$, y medio pero por encima de 0,7 en cuatro variables $(d=0,737$ para CFI; $d=0,722$ para LPT; $d=0,785$ para LPSA; $d=0,750$ para IFL). Del resto de variables, la que presentó un tamaño del efecto más pequeño es RIT $(d=0,509)$.

\section{Tabla 3}

Estadísticos descriptivos, prueba t, nivel de significación, Potencia observada ( $\beta$ ) y tamaño del efecto $(d)$

\begin{tabular}{llrrcccc}
\hline Variable & Medida & \multicolumn{1}{c}{$M$} & \multicolumn{1}{c}{$D T$} & \multicolumn{1}{c}{$t$} & \multicolumn{1}{c}{$p$} & $d$ \\
\hline RLA & PRE & 35,13 & 1,585 & $-4,043$ & 0,000 & 0,996 & 0,519 \\
& POST & 35,87 & 0,412 & & & & \\
RLT & PRE & 48,24 & 18,162 & 8,040 & 0,000 & 0,999 & 0,682 \\
& POST & 37,21 & 12,767 & & & & \\
CFI & PRE & 8,87 & 1,530 & $-5,165$ & 0,000 & 0,999 & 0,737 \\
& POST & 9,85 & 0,669 & & & & \\
CFD & PRE & 5,79 & 2,518 & $-8,850$ & 0,000 & 1,000 & 1,000 \\
& POST & 8,07 & 1,922 & & & & \\
LPA & PRE & 15,46 & 5,231 & $-6,484$ & 0,000 & 0,999 & 0,803 \\
& POST & 19,18 & 1,667 & & & & \\
LPT & PRE & 98,18 & 83,546 & 6,241 & 0,000 & 0,999 & 0,722 \\
& POST & 44,56 & 25,031 & & & & \\
LPSA & PRE & 7,46 & 3,098 & $-6,008$ & 0,000 & 0,999 & 0,785 \\
\multirow{2}{*}{ LPST } & POST & 9,62 & 0,947 & & & & \\
& PRE & 37,13 & 27,646 & 5,286 & 0,000 & 0,999 & 0,619 \\
RIT & POST & 22,30 & 13,563 & & & & \\
& PRE & 27,65 & 9,294 & 5,934 & 0,000 & 0,995 & 0,509 \\
IFL & POST & 23,31 & 7,448 & & & & \\
& PRE & 40,02 & 32,404 & $-11,55$ & 0,000 & 0,999 & 0,750 \\
& POST & 64,74 & 33,455 & & & & \\
\hline
\end{tabular}

Nota: RLA = aciertos de denominación rápidamente de letras escritas, $\mathrm{RLT}=$ tiempo de denominación rápidamente de letras escritas tiempo, $\mathrm{CFI}=$ conciencia fonémica de sonido inicial, CFD = conciencia fonémica de sonido diferente, LPA = lectura de palabras aciertos, LPT = tiempo de lectura de palabras, LPSA = Lectura de pseudopalabras aciertos, LPST = tiempo de lectura de pseudopalabras, RIT = tiempo de denominación rápida de imágenes , IFL = Índice de fluidez léxica, $\mathrm{PRE}=$ Pretest, $\mathrm{POST}=$ Postest

En la Tabla 4 se observa el número de sujetos en cada uno de los niveles, así como la puntuación media (M) y la desviación típica (DT) en dichos niveles. Respecto al pretest, con este criterio, el riesgo se redujo a un sujeto que se consideró resistente al aprendizaje y fue derivado a una evaluación más específica de la lectura. 


\section{Tabla 4}

Número de sujetos de cada grupo (n), media (M) y la desviación típica (DT)

\begin{tabular}{lccc}
\hline Niveles & $\mathrm{N}$ & $\mathrm{M}$ & $\mathrm{DT}$ \\
\hline Establecido & 15 & 111,08 & 33,553 \\
Emergente & 55 & 43,80 & 14,905 \\
Riesgo & 1 & 6,94 & - \\
\hline
\end{tabular}

Una vez terminada la propuesta se revisó el criterio de acuerdo con los nuevos resultados para continuar trabajando con la finalidad de compensar las diferencias iniciales y reducir el riesgo lector desde el aula. Dado que el aprendiz resistente ya fue derivado, para establecer el criterio 2 no fue considerado. De nuevo, aquellos participantes cuyo tiempo estaba por debajo del percentil $20(32,258)$ deberían formar parte directamente del nivel de Riesgo y no se utilizarían para calcular la medida de corte. En este caso, el criterio 2 fue establecido por una media de 64,74 y una desviación típica de 33,455 para el IFL; así, aquellos sujetos cuyas puntuaciones estaban una desviación típica por debajo de la media pasarían a formar parte del nivel de Riesgo $(<31,29)$, los que obtuviesen puntuaciones que se encontraban una desviación típica por encima de la media formarían el nivel Establecido $(>98,2)$. Todos aquellos cuyas puntuaciones se encontraban entre los dos valores anteriores formarían el nivel Emergente (entre 98,19 y 31,30).

En la Tabla 5 se observa el número de sujetos que estaban en los tres grupos, así como la media y la desviación típica del IFL de los grupos después del entrenamiento.

\section{Tabla 5}

Número de sujetos de cada grupo (n), media (M) y la desviación típica (DT)

\begin{tabular}{lccc}
\hline Niveles & $\mathrm{n}$ & $\mathrm{M}$ & $\mathrm{DT}$ \\
\hline Establecido & 9 & 131,94 & 26,933 \\
Emergente & 50 & 52,65 & 15,098 \\
Riesgo & 12 & 21,82 & 6,484 \\
\hline
\end{tabular}

El ANOVA de un factor entre niveles en la variable IFL después de implementar la propuesta muestra efectos significativos en los tres niveles $(F(2,68)=129,312$; $\mathrm{p}<0,001)$. En la prueba post hoc de Scheffé se siguen observando diferencias estadísticamente significativas entre el nivel Establecido y el Emergente $(p<0,001)$, entre el Establecido y el Riesgo $(\mathrm{p}<0,001)$, y entre el nivel Emergente y el de Riesgo $(\mathrm{p}<0,001)$.

Se debe valorar también la evolución del alumnado por los niveles, especialmente del alumnado catalogado de riesgo en el pretest. Así, se pudo describir la trayectoria individual de cada aprendiz de acuerdo con el criterio del grupo. Hubo un grupo de participantes (11) que incrementó su nivel de rendimiento: 3 participantes pasaron del nivel Emergente al nivel Establecido y 8 del nivel de Riesgo al de Emergente. 
También se observó la tendencia contraria. En ese caso 7 participantes pasaron del nivel Emergente al de Riesgo y 1 participante del nivel Establecido al Emergente.

Después de haber implementado la propuesta, la distribución de los participantes tomando como variables el tiempo (LPT) y el número de aciertos en lectura de palabras (LPA) era menos diversa, como se puede observar en la Figura 2. En la Tabla 3 se recogen los estadísticos descriptivos para cada prueba. El grupo era más homogéneo y se redujeron tanto el riesgo y como la diversidad. Se apreció un patrón de ejecución estable que supone que se siguen manteniendo en POST diferencias significativas entre los niveles (Aguilar et al., 2017; Pfost et al., 2014). Un ejemplo paradigmático de esta evolución es la trayectoria del participante 26 quien en el pretest leyó 15 palabras correctamente en 40 segundos y en POST leyó las 20 palabras correctamente en 28 segundos.

\section{Figura 2}

Distribución final de los sujetos en función del tiempo y lectura correcta de palabras

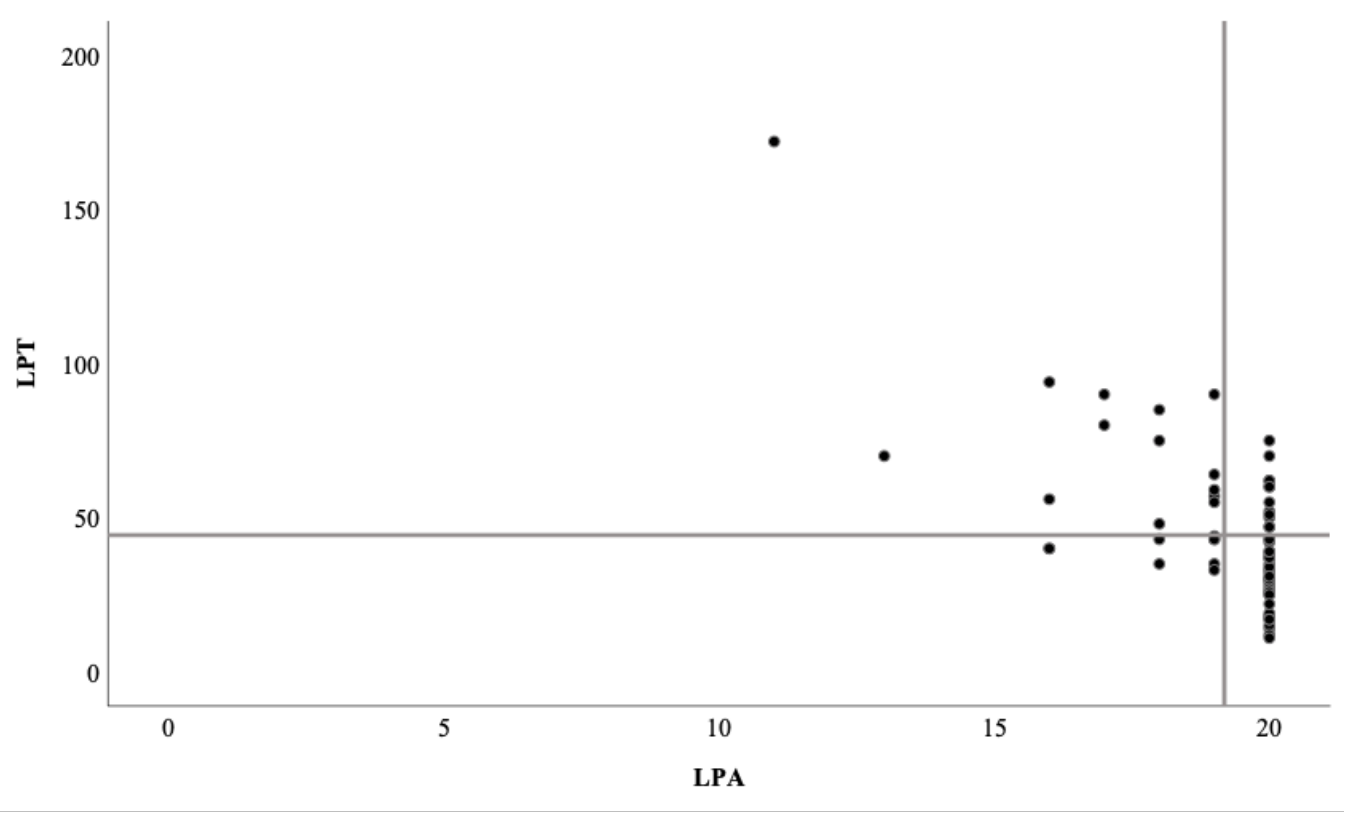

\section{Discusión}

En este trabajo se presenta la aplicación de una propuesta para desarrollar el conocimiento profesional del profesorado en la prevención y la atención al alumnado en situación de riesgo en el aprendizaje de la lectura. Se discute la adecuación de la propuesta para explicitar el conocimiento profesional, mejorar el sistema de respuesta del profesorado en el aula y compensar la diversidad inicial del alumnado (Didion et al., 2019; Mena et al., 2008; Pozo y Gómez-Crespo, 2005; Sánchez y Mena, 2010; Scarparolo y Hammond, 2018; Schön, 1998). 
Para explicitar el conocimiento profesional, de acuerdo con el modelo constructivista de asesoramiento (García et al., 2003; Sánchez, 2001), se confirmó la utilidad de iniciar el asesoramiento y la formación del profesorado mediante un diálogo abierto con el fin de identificar lo que hace en el aula, las dificultades que encuentra y su motivación y percepción de competencia en cuanto a la enseñanza de la lectura. La voluntariedad de la participación y el buen clima de convivencia que imperaba en los centros facilitó la colaboración.

Respecto a la mejora de la calidad de la instrucción a partir de la reflexión inicial sobre la práctica de enseñanza del lenguaje escrito (García et al., 2003; Sánchez, 2000; Sánchez y Mena, 2010), se propuso un seguimiento quincenal de la práctica para garantizar la integración de las propuestas en el sistema de respuesta del profesorado venciendo así la tendencia al abandono o falta de continuidad en el seguimiento de la misma que refiere la literatura (Mena et al., 2008; Snow et al., 2005). Tal y como expresa una maestra en el trabajo de McCutchen et al. (2002) "Lo que hago ahora no es muy distinto de lo que hacía antes. Pero lo que es diferente ahora es que tengo una idea clara de lo que quiero que mi alumnado aprenda exactamente. Ahora estoy más centrada en el aprendizaje y mis estudiantes parecen también más centrados". (p. 13).

Los resultados permitieron a las maestras confirmar en su propio grupo-clase el funcionamiento de lo que en las investigaciones ya se ha evidenciado respecto a los precursores de la lectura en castellano (Aguilar et al., 2017; Defior \& Serrano, 2011; Suárez-Coalla et al., 2014), a la identificación de palabras (Álvarez-Cañizo et., 2017; Ehri, 2005; Samuels, 1979; Suárez-Coalla et al., 2014) y a los niveles operativos en la enseñanza de la fluidez (Good et al., 2001; Samuels,1979).

\section{Conclusiones}

Algunas claves del procedimiento se presentan a modo de conclusión:

1. Establecer la prevención y la reducción de la diversidad y del riesgo lector como un reto colectivo y concreto. El reto fue asumido por el profesorado que se comprometió a reducir el riesgo a corto y largo plazo. Además, ese compromiso del profesorado se formuló de una manera concreta tras el análisis grupal de los resultados del pretest. Su pretensión era reducir el alumnado de riesgo y lograr que no se obtuvieran diferencias significativas tras la intervención (Aguilar et al., 2017; Pfost et al., 2014).

2. En la intervención en el aula se dio autonomía al profesorado que se vio abocado a actuar y a asumir el liderazgo. Este aspecto fue determinante para favorecer el desarrollo profesional. Scanlon et al. (2008) mostraron que este tipo de intervención era más efectiva para reducir el riesgo que una intervención en la que participaba un experto como asesor en el aula. La razón es que el profesorado que combinaba su actuación con la de un experto no asumía su acción con el mismo grado de compromiso y motivación.

3. El modelo RTI representó una oportunidad para desarrollar la motivación y la percepción de competencia entre el profesorado. Aunque no se midió con una prueba específica en la evaluación final, el profesorado atribuyó a la calidad de la instrucción que había puesto en marcha en el aula la mejoría en el rendimiento lector del 
alumnado, la reducción del grupo de riesgo y el establecimiento de un patrón de ejecución estable. Este aspecto se recoge en los comentarios finales del profesorado sobre la experiencia. Señalaron, además, que la propuesta resultó interesante porque permitió superar lo que una maestra denominó “temor a la diversidad”. Asimismo, valoraron positivamente el sentido dinámico con que se caracterizó del riesgo lector porque reflejaba el trabajo en el aula y lo alejaba de una categoría diagnóstica propia de especialistas.

4. La presentación y discusión de los resultados en el grupo de trabajo facilitó que se minimizara la frustración derivada de la no consecución en el postest de la meta planteada. Esto es, a pesar del esfuerzo realizado no se compensaron las diferencias iniciales, ya que las diferencias entre los niveles, aunque se redujeron, siguieron siendo significativas. El análisis colectivo permitió profundizar en la dimensión teórica y práctica de la experiencia para concluir que era necesario darle continuidad implicando al menos al profesorado de $1^{1}$ y y $2^{\circ}$ de Educación Primaria. Se subrayó que la compensación de las diferencias es una tarea colectiva que demanda tiempo y dedicación. La continuidad de la propuesta se vio frustrada por la crisis sanitaria del COVID.

5. La aplicación exitosa de la propuesta no permite, sin embargo, establecer una evidencia sobre la misma como una modalidad formativa más eficaz que otras. Simplemente se puede afirmar que ha funcionado en el contexto en que se realizó y ha resultado satisfactoria para el profesorado. Para validar esta propuesta a partir de esta experiencia inicial se planteará un diseño metodológico más exigente en el que se compare su eficacia con otro tipo de intervenciones o con un grupo de control (Didion et al., 2019). En este sentido la limitación de este trabajo lo circunscribe a una condición de estudio preliminar que debe ser confirmada.

\section{Agradecimientos}

Esta investigación se ha desarrollado dentro del proyecto financiado PAPI-17-EMERG-49 de la Universidad de Oviedo.

\section{Conflicto de intereses}

Los autores declaran no tener ningún conflicto de intereses. Los financiadores no tuvieron ningún papel en el diseño del estudio; en la recopilación, análisis o interpretación de datos; en la redacción del manuscrito, o en la decisión de publicar los resultados.

\section{Referencias}

Aaron, P.G., Joshi, R.M., Gooden, R., \& Bentum, K.E. (2008). Diagnosis and treatment of reading disabilities based on the component model of reading: An alternative to the discrepancy model of LD. Journal of Learning Disabilities, 41(1), 67-84. doi: $10.1177 / 0022219407310838$.

Acosta, V.M., Moreno, A.M., Axpe, M.Á., \& Quintana, A. (2008). La identificación de barreras para el aprendizaje de la lectura entre alumnado de riesgo desde una perspectiva integrada. Revista de Logopedia, Foniatría y Audiología, 28(4), 231244. doi: 10.1016/S0214-4603(08)70130-X 
Aguilar, M., Aragón, E., Navarro, J.I., Delgado, C., \& Marchena, E. (2017). Análisis del efecto san Mateo en un estudio longitudinal sobre el desarrollo lector durante la

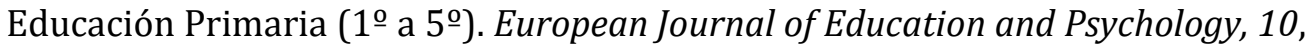
23-32. https://doi.org/10.1016/j.ejeps.2016.07.001

Alegría, J. (2006). Por un enfoque psicolingüístico del aprendizaje de la lectura y sus dificultades. 20 años después. Infancia y Aprendizaje, 1, 93-111. https://doi.org/10.1174/021037006775380957

Álvarez-Cañizo, M., Suárez-Coalla, P., \& Cuetos, F. (2017). Reading prosody development in Spanish children. Reading and Writing: An Interdisciplinary Journal, 31(1), 35-52. https://doi.org/10.1007/s11145-017-9768-7

Arrow, A.W., Braid, C., \& Chapman, J.W. (2019). Explicit linguistic knowledge is necessary, but not sufficient, for the provision of explicit early literacy instruction. Annals of Dyslexia, 69, 99-113. http://dx.doi.org/10.1007/s11881018-00168-0

Castejón, L., González-Pumariega, S., \& Cuetos, F. (2015). Desarrollo de la fluidez en la lectura de palabras en Educación Primaria: un seguimiento longitudinal de seis años. Infancia y Aprendizaje, 38, 842-871. https://doi.org/10.1080/02103702.2015.1076266

Cohen, J. (1988). Statistical power analysis for the behavioral sciences (2 ${ }^{\mathrm{a}}$ Ed.). Academic Press.

Defior, S., \& Serrano, F. (2011). La conciencia fonémica, aliada de la adquisición del lenguaje escrito. Revista de Logopedia, Foniatría y Audiología, 31(1), 2-13. https://doi.org/10.1016/S0214-4603(11)70165-6

Didion, L., Toste, J.R., \& Filderman, M.J. (2019). Teacher professional development and student reading achievement: A meta-analytic review of the effects. Journal of Research on Educational Effectiveness, 13(1), 29-66. http://dx.doi.org/10.1080/19345747.2019.1670884

Ehri, L.C. (2005). Learning to read words. Theory, findings and issues. Scientific Studies of Reading, 9, 167-188.

Evens, M., Elen, J., Larmuseau, C., \& Depaepe, F. (2018). Promoting the development of teacher professional knowledge: Integrating content and pedagogy in teacher education. Teaching and Teacher Education, 75, 244-258. https://doi.org/10.1016/j.tate.2018.07.001

García, J.R., Rosales, J., \& Sánchez, E. (2003). El asesoramiento psicopedagógico como construcción de significados compartidos: un estudio sobre su dificultad. Cultura y Educación, 15(2), 129-148.

DOI: $10.1174 / 113564003770717431$

González-Seijas, R.M., \& Cuetos, F. (2008). ALE, actividades, aprendizaje, lectura y escritura. CEPE.

Good, R.H., Simmons, D., \& Kame'enui, E. (2001). The importance and decision making utility of a continuum, of fluency-based indicators of foundational readings kills for third-grade high-stakes outcomes. Scientific Studies of Reading, 5, 257-288. doi: 10.1207/S1532799XSSR0503_4

Gutiérrez-Fresneda, R., \& Díez-Mediavilla, A. (2015). Aprendizaje de la escritura y habilidades de conciencia fonológica en las primeras edades. Bordón. Revista de 
Pedagogía, 67(4), 43-59. https://doi.org/10.13042/Bordon.2015.67405

Jiménez, J.E. (2019). El modelo de respuesta a la intervención. Un enfoque preventivo para el abordaje de dificultades específicas de aprendizaje. Pirámide.

Jiménez, J.E., \& Ortiz, M.R. (1995). Conciencia fonológica y aprendizaje de la lectura: teoría, evaluación e intervención. Síntesis

Jiménez, J.E., Rodríguez, C., Suárez, N., O’Shanahan, I., Villadiego, Y., Uribe, C., Villalobos, J.A., \& Rodas, P. (2015). Teachers' implicit theories of learning to read: A crosscultural study in Ibero-American countries. Reading and Writing, 28(9), 13551379. Doi:10.1007/s11145-015-9574-z

Lane, H.B., Hudson, R.F., Leite, W.L., Kosanovich, M.L., Taylor, M., Fenty, N.S., \& Wright, T. (2009). Teacher knowledge about reading fluency and indicators of students' fluency growth in reading first schools. Reading and Writing Quartely, 25, 5786. https://doi.org/10.1080/10573560802491232

Ledesma, R., Macbeth, G., \& Cortada, N. (2008). Tamaño del efecto: revisión teórica y aplicaciones con el sistema estadístico. ViSta. Revista Latinoamericana de Psicología, 40(3), 425-439.

McCutchen, D., Abbott, R.D., Green, L.B., Beretvas, S.N., Cox, S., Potter, N.S., Quiroga, T., \& Gray, A.L. (2002). Beginning literacy: links among teacher knowledge, teacher practice, and student learning. Journal of Learning Disabilities, 35(1), 69-86. doi: 10.1177/002221940203500106.

Mena, J.J., Sánchez, E., \& Tillema, H. (2008). Teachers reflecting on their work: Articulating what is said about what is done. Teachers and Teaching Theory and Practice, 14(2), 95-114. doi: 10.1080/13540600801965887

National Professional Development Center on Inclusion (NPDCI) (2008). What do we mean by professional development in the early childhood field? Chapel Hill: University of North Carolina, FPG Child Development Institute.

Osman, D.J., \& Warner, J.R. (2020). Measuring teacher motivation: The missing link between professional development and practice. Teaching and Teacher Education, 92, 103064. doi:10.1016/i.tate.2020.103064

Ou, S.R., Mersky, J.P., Reynolds, A.J., \& Kohler, K.M. (2007). Alterable predictors of educational attainment, income, and crime: Findings from an inner-city cohort. Social Service Review, 81(1), 85-128. https://doi.org/10.1086/510783

Pozo, J.I., \& Gómez-Crespo, M.A. (2005). The embodied nature of implicit theories: The consistency of ideas about the nature of matter. Cognition and Instruction, 23(3), 351-387. https://www.jstor.org/stable/3568095

Pfost, M., Hattie, J., Dörfler, T., \& Artelt, C. (2014). Individual differences in reading development: A review of 25 years of empirical research on Matthew effects in reading. Review of Educational Research, 44, 402-420. http://dx.doi.org/10.1177/0022219411417568.

Sammuels, S.J. (1979). The method of repeated readings. The Reading Teacher, 32, 403408.

Sánchez, E. (2000). El asesoramiento psicopedagógico: un estudio observacional sobre las dificultades de los psicopedagogos para trabajar con los profesores. Infancia y Aprendizaje, 91, 55-78. doi: 10.1174/021037000760087865

Sánchez, E. (2001). Ayudando a ayudar: el reto de la investigación educativa. Cultura y 
Educación, 13(3), 249-266. https://doi.org/10.1174/113564001753207582

Sánchez, E., García, R., \& Rosales, J. (2010). La lectura en el aula. Qué se hace, qué se debe hacer y quénse puede hacer. Graó.

Sánchez, E., \& Mena, J.J. (2010). Hablamos de lo que no existe; y de lo que existe, no hablamos. Infancia y Aprendizaje, 33(2), 185-197. https://doi.org/10.1174/021037010791114661

Scanlon, D.M., Gelzheiser, L.M., Vellutino, F.R., Schatschneider, C., \& Sweeney, J.M. (2008). Reducing the incidence of early reading difficulties: Professional development for classroom teachers vs. direct interventions for children. Learning and Individual Differences, 18(3), 346359. doi: $10.1016 /$ j.lindif.2008.05.002

Scanlon, D.M., Vellutino, F.R., Small, S.G., Fanuele, D.P., \& Sweeney, J. (2005). Severe reading difficulties: Can they be prevented? A comparison of prevention and intervention 209-227. doi: $10.1207 /$ s15327035ex1304_3

Scarparolo, G.E., \& Hammond, L.S. (2018) The effect of a professional development model on early childhood educators' direct teaching of beginning reading. Professional Development in Education, 44(4), 492506. doi: 10.1080/19415257.2017.1372303

Schön, D. (1998). El profesional reflexivo: cómo piensan los profesionales cuando actúan. Paidós.

Sellés, P., Martínez, T., \& Vidal-Abarca, E. (2010). Batería de Inicio a la Lectura (BIL 36): diseño y características psicométricas. Bordón. Revista de pedagogía, 62(2), 137-160.

Snow, C.E., Griffin, P.E., \& Burns, M. (Eds.) (2005). Knowledge to support the teaching of reading: Preparing teachers for a changing world. Indianapolis: Jossey-Bass.

Suárez-Coalla, P., Álvarez-Cañizo, M., \& Cuetos, F. (2014). Orthographic learning in Spanish children. Journal of Research in Reading, 39, 292-311. https://doi.org/10.1111/1467-9817.12043

Valencia, S.W., Smith, T.A., Reece, A.M., Li, M., Wixson, K.K., \& Newman, H. (2010). Oral reading fluency assessment: Issues of construct, criterion, and consequential validity. Reading Research Quarterly, 45, 270291. https://doi.org/10.1598/RRQ.45.3 